\title{
Analysis of Image Visibility in Projection-type Integral Imaging System without Diffuser
}

\author{
Soon-gi Park, Byoung-Sub Song, and Sung-Wook Min* \\ Department of Information Display, Kyung Hee University, \\ 1 Hoeki-dong, Dongdaemoon-gu, Seoul 130-701, Korea
}

(Received January 6, 2010 : revised March 24, 2010 : accepted April 2, 2010)

\begin{abstract}
We analyze image visibility of a projection-type integral imaging system without diffuser, in terms of the fill factor, which is determined by the relationship between the exit pupil of the projection system and the size and the focal length of the elemental lens. High fill factor is a requirement for good visibility. Moreover, for psychological reasons, for the same fill factor, better visibility is accomplished using a relatively small elemental lens. In this paper, we study image visibility through basic experiments and results.
\end{abstract}

Keywords: Integral imaging, Fill factor, Projection-type, Visibility

OCIS codes : (110.2990) Image formation theory; (100.6890) Three-dimensional image processing

\section{INTRODUCTION}

Lippmann proposed integral imaging, three-dimensional (3D) display technology which shows full parallax images and does not need any additional equipment[1]. Recently, integral imaging systems have improved rapidly thanks to the progress of flat panel displays such as liquid crystal displays (LCD), which allow the expression of full color 3D moving pictures with a continuous viewing zone.

But realizing a high quality integral imaging system requires a tremendous number of pixels because of the tradeoff relationships between display resolution and viewing parameters of the integrated image. These parameters include imaging resolution, viewing angle and imaging depth[2]. In order to overcome the problem of spatial limitation of resolution, several kinds of integral imaging systems which show great improvement of viewing qualities by using multiple display devices or multiple imaging planes, were proposed [3-7],

Projection-type display is frequently used as a display device for elemental images in an integral imaging system [7-12]. For a projection-type integral imaging system, it is easy to control the size of the image and the pixel pitch, and a large size screen is achieved simply through system expansion using a multi-vision method.

An ordinary projection-type integral imaging system needs a diffuser screen, on which elemental images are located. Employing the diffuser screen incurs some disadvantages, because the resolution of the elemental image is restricted due to the diffusing grain, and optical light loss reduces the optical power efficiency of the system. Previous research, used some kind of projection-type system without diffuser screen[11, 12]. Those systems adopted a collimating lens with large aperture or a lens array with small elemental lens pitch, to test the feasibility of a system without diffuser.

The previous studies, however, did not explain the exact relationship between the visibility and the system configuration. The relationship between the visibility and each component of the system can be a guideline for designing a projection-type integral imaging system for various applications.

In this paper, we analyze the relationship between the exit pupil of a projection lens system and the elemental lenses, according to their geometrical shape and position. This relationship can be simply described as a fill factor, which represents the ratio of the effective imaging area to the area of the elemental lens. Moreover, for psychological reasons, the configuration of the lens array, especially the size of the elemental lens, also affects visibility. We show briefly the relation between visibility and psychological factors. Some basic experiments and results are presented.

\footnotetext{
*Corresponding author: mins@khu.ac.kr

Color versions of one or more of the figures in this paper are available online.
} 


\section{FILL FACTOR}

\subsection{Fill factor of central lens}

The fill factor is the ratio of the effective imaging area and the area of each individual elemental lens. In the projection-type integral imaging system without diffuser, the visibility of the reconstructed image is greatly affected by the fill factor. In this system, the exit pupil of the projection lens is observed through the elemental lens, and the image inside of the exit pupil made by the elemental lens becomes the effective image. The fill factor depends on the geometry of the elemental lens and the shape of the aperture stop in the projection lens, which means we need a different formula to calculate the fill factor for different shapes of elemental lens or of projection lens aperture stop.

The fill factor calculation can be divided into two cases. For the first case, the image of the exit pupil of the projection lens is larger than the elemental lens; the second case is the opposite case. If the image of the exit pupil is larger than the elemental lens, the fill factor must be full. For the other case, we need to consider the geometry of the aperture stop and the elemental lens.

The image radius of the exit pupil of the projection lens system through the elemental lens, $r_{\text {image }}$, defined from the geometry as shown in Fig. 1, can be expressed as:

$$
r_{\text {image }}=\frac{r_{\text {pupil }} f}{d-f}
$$

where, $r_{p u p i l}$ is the radius of the exit pupil of the projection lens system, $d$ is the distance between the exit pupil and the elemental lens, and $f$ is the focal length of the elemental lens. We assume that the projection lens has a circular exit pupil and that the elemental lens has square geometry. For this case, the fill factor becomes as follows:

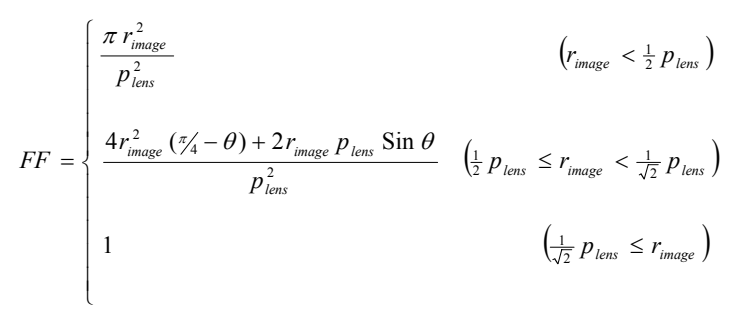

where $F F$ is the fill factor, $p_{\text {lens }}$ is the elemental lens pitch,

$$
\text { and } \theta=\cos ^{-1} \frac{p_{\text {lens }}}{2 r_{\text {pupil }}} \text {. }
$$

Figure 2 shows the value of fill factor as a function of the size and the focal length of the elemental lens when $r_{\text {pupil }}$ is $30 \mathrm{~mm}$ and $d$ is $300 \mathrm{~mm}$.

As shown in Fig. 2, we can assume that the elemental lens size is the most critical factor for achieving a higher fill factor because of the limitations on manufacturing an

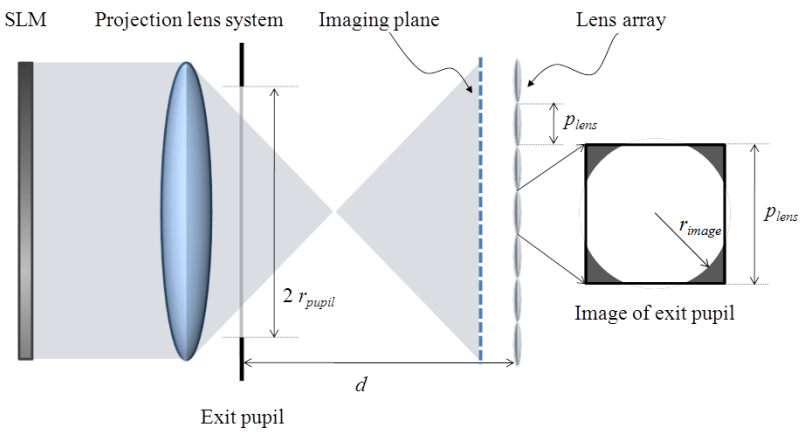

FIG. 1. Schematic diagram of projection-type integral imaging system without diffuser.

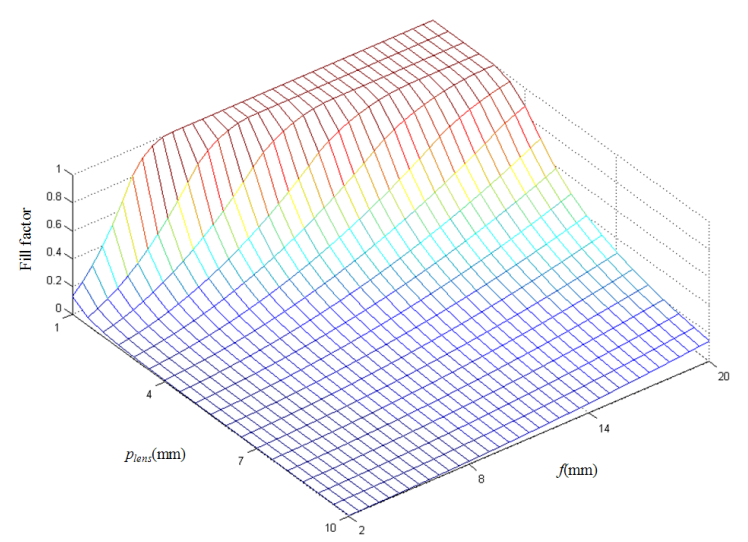

FIG. 2. Fill factor according to lens pitch $p_{\text {lens }}$ and focal length $f$.

elemental lens with short focal length and large aperture simultaneously. Actually, in Fig. 2, the region where the $\mathrm{f}$ number, the ratio between focal length and lens pitch, becomes less than 1 is practically impossible to achieve.

\subsection{Overall fill factor}

The fill factor is also decided by the position of the elemental lens on the imaging plane. Because the integral imaging system is composed of a lens array, the calculation of fill factor for each elemental lens is necessary. Overall fill factor is defined by the sum of the whole effective area divided by the area of the display device (or the area of the entire lens array). It is equal to the average value of each fill factor as shown in eq. (3).

$$
\begin{aligned}
{[\text { Overall fill factor] }} & \equiv \frac{[\text { Effective image area] }}{[\text { Lens array area }]} \\
& =\frac{\sum_{m=1}^{M} \sum_{n=1}^{N} p_{\text {lens }}^{2} F F_{m n}}{M N p_{\text {lens }}^{2}}=F F_{\text {average }}
\end{aligned}
$$

where, $M$ and $N$ represent the number of horizontal and vertical elemental lenses, $F F_{m n}$ is the fill factor of the $\mathrm{m}^{\text {th }}$ horizontal and the $\mathrm{n}^{\text {th }}$ vertical elemental lens, and $F F_{\text {average }}$ 
is the average fill factor of the entire lens array. When it comes to the lens array, we need to consider the movement of the imaging area, or simply the shifting, which causes the vignetting of the image as a result of the shifted position of the elemental lens. For each elemental lens, the shifting can be calculated from its position, which can be decided by the distance from the optical axis of the projection lens system.

The shifting distance of the pupil image depends on the magnification and the pitch of the elemental lens. Needless to say, it depends on the position of the elemental lens as well. The shifting can be easily calculated from the similarity of triangles made by the exit pupil of the projection lens system and the image of the exit pupil through the elemental lens.

We assumed that the elemental lens which is located in the center of the lens array has no shifting of image. This elemental lens is square shaped, and the elemental lenses are aligned without a gap. Then the shifting of the exit pupil image through the elemental lens which is a neighbor to the central lens is expressed as the following eq. (4).

$$
S=\frac{[\text { Size of lens }]}{[\text { Magnification }]}=\frac{p_{\text {lens }} f}{|f-d|}
$$

where $S$ is the shifting distance of the exit pupil image. The lens which is located $\mathrm{m}$ lenses horizontally and $n$ lenses vertically apart from the center has $m$ times the horizontal and $n$ times the vertical shifting compared to the first neighbor lenses.

If the shift is very large so that the image is located beyond the elemental lens boundary, the fill factor becomes zero. On the other hand, if the shift is small, so that it fulfills the inequality in formula (5), the fill factor remains the same as the fill factor of the central lens.

$$
S<\frac{1}{2} p_{\text {lens }}-r_{\text {image }}
$$

Usually, when the distance $d$ is large enough to satisfy

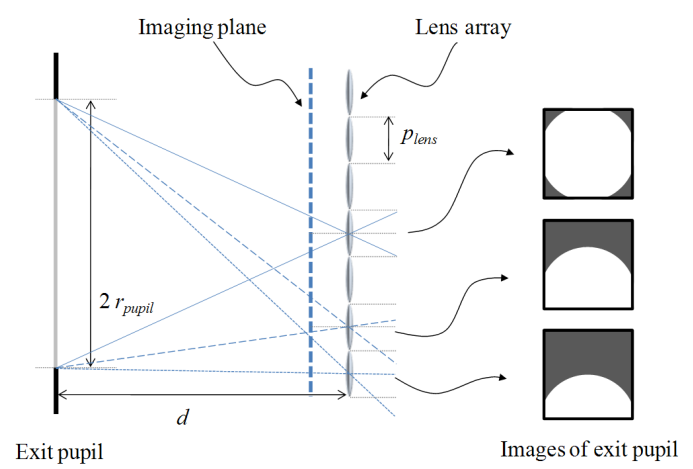

FIG. 3. Shifting of exit pupil image through elemental lens. the inequalities in formula (6), the shift at the outmost elemental lens does not invade the border of the elemental lens, and the overall fill factor is not different from the fill factor of the central lens.

$$
\frac{m\left(3 p_{\text {lens }}+2 r_{\text {pupil }}\right) f}{p_{\text {lens }}}<d \frac{n\left(3 p_{\text {lens }}+2 r_{\text {pupil }}\right) f}{p_{\text {lens }}}<d
$$

Of course, the shift of pupil image due to the position of the elemental image is affected by the geometric arrangement of the elemental lenses. For a more general case, such as an arbitrarily shaped aperture stop with hexagonal or triangular elemental lens configuration, the calculation of overall fill factor will be complex.

For this reason, we propose a computational approach to get the overall fill factor. From the definition, the overall fill factor is defined by the ratio of the effective imaging area and the lens array area. Getting the effective image area is quite similar to the pickup process in integral imaging. Simply, the exit pupil can be regarded as the object and the corresponding elemental image can be considered as the observed image in the projection-type integral imaging system without diffuser. These kinds of images can be easily obtained by the computer-generated integral imaging method. The ratio of the number of pixels inside and outside of the boundary of the exit pupil gives the overall fill factor. Using this technique, the fill factor can be calculated simply even if the exit pupil of the projection lens system and the elemental lens have a complicated geometry.

\section{VISIBILITY ANALYSIS}

If the fill factor of the system is equal to unity, there is no difference in the visibility compared to the conventional integral imaging system. On the other hand, the visibility of the image is gradually degraded as the fill factor decreases. As shown in Fig. 4, however, even though the images have the same fill factor, each image can show different visibilities. The image composed of a larger number of elemental image shows better visibility. This is because of the psychological factor which can be explained by Recognition-By-Component

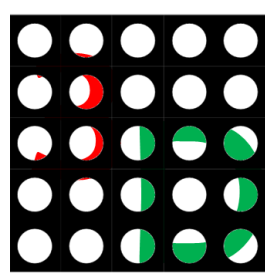

(a)

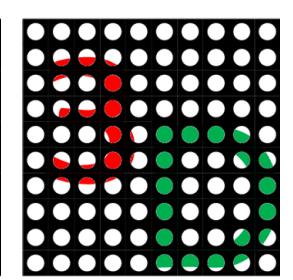

(b)

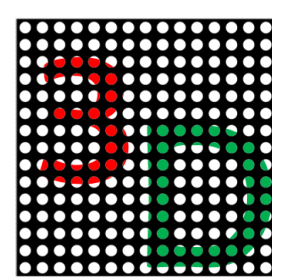

(c)
FIG. 4. Comparison of visibility with simulated result for different configuration of lens array with same fill factor(0.3). (a) $5 \times 5$ lenses, (b) $10 \times 10$ lenses, (c) $15 \times 15$ lenses. 
theory(RBC theory)[13]. Although the examples shown in Fig. 4 are two-dimensional images, which are viewpoint independent, $\mathrm{RBC}$ theory can be directly applied in $3 \mathrm{D}$ images of the integral imaging system[14].

According to RBC theory, a person recognizes the object as a combination of simple three-dimensional objects called geons(geometrical icons). Each geon has simple characteristics, for example the collinearity of line or the termination of curves at a common point. And those characteristics discriminate the geons from other geons. RBC theory states that if the geons of an object and the arrangement of the parts can be perceived, the whole object can be identified even when the images are occluded or degraded.

Because the geons are very simple objects, the clues through small apertures are enough to allow recognition of geons. Consequently, a lens array that consists of a large number of elemental lenses has more chance to show the clues for geons, resulting in better visibility. Besides, a smaller elemental lens provides a higher fill factor according to the analysis of section II. However, if the lens pitch or the size of the exit pupil is reduced under the pixel pitch of the elemental images, the system operates as if it is operated in a focused mode of a conventional integral imaging system. In a focused mode, the number of elemental lenses is identical to the number of $3 \mathrm{D}$ image pixels and the image recognition process becomes very similar to the case for $2 \mathrm{D}$ images.

In order to compare the quality of the systems in a quantitative manner, we express the total number of pixels in terms of the number of lens in an array and the fill factor, which will provide the most accurate criterion for comparison between systems. The total number of pixels is given by eq. (7).

$$
N_{\text {pixel }}=\left(\frac{p_{\text {lens }}}{p_{\text {pixel }}}\right)^{2} \times F F_{\text {average }} \times N_{\text {lens }}
$$

where, $N_{\text {pixel }}$ refers to the total number of $p_{\text {ixels, }}$, ppixel is a pixel pitch of the display device, and $N_{\text {lens }}$ stands for the total number of elemental lenses in a lens array. The first term on the right side stands for pixel density which is related to the expression of $2 \mathrm{D}$ texture resolution of the image, while the fill factor and total number of lenses are important for recognition of 3D objects. Equation 7 also implies the trade-off relationship between texture quality and object recognition.

In addition to the configurations of the lens array, the visibility also depends on the condition of the observer or the contents of the reconstructed image. Especially when the observer or the reconstructed image is in motion, the chance of exposing the clues of geons increases.

Consequently, the visibility of an integrated image is greatly affected by the relative size of objects compared to the lens pitch of the elemental lens, because the opportunity for perceiving geons is decided by the number of elemental lenses.

\section{EXPERIMENT}

We compared the experimental results of three different lens arrays with and without the diffuser in order to test our analysis. Figure 5 shows the schematic diagram of the experimental setup. The distance $d$ between exit pupil and lens array is the sum of the projection distance, $d_{\text {projection }}$ and the gap, g between imaging plane and lens array. The projection distance depends on the specification of the projection lens, and the gap is mainly affected by the focal length of the elemental lens.

In the experiment, we set the projection distance much longer than the focal length of the elemental lens in order to keep the shift small. Therefore, we can directly calculate the overall fill factor from the given specifications of system. Besides, with small shifting, we can adjust the system setting to get the desired fill factor without complicated calculation.

We use the typical LCD monitor with luminance of 200 $\mathrm{cd} / \mathrm{m}^{2}$. And, we adopt a single biconvex lens as a projection lens for simplicity. We put the aperture stop in front of the projection lens. The aperture also plays the role of the exit pupil of the projection lens. The projection distance from the exit pupil of the projection lens system to the imaging screen is around $340 \mathrm{~mm}$. The size of the projected image is $60 \mathrm{~mm} \times 60 \mathrm{~mm}$. In the system, we adjust the radius of aperture in the projection lens so that we can obtain the same fill factor for the three different configurations of lens arrays. Table 1 shows the specifications of lens arrays used in the experiment.

For the experiment, we set the target fill factor at 0.3 and the corresponding radii of the exit pupil of the projection lens system for $10 \mathrm{~mm}, 5 \mathrm{~mm}$ and $1 \mathrm{~mm}$ elemental

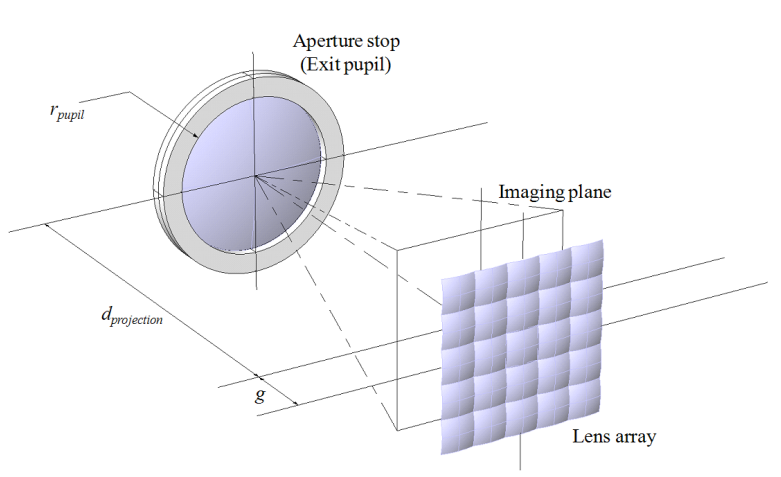

FIG. 5. Schematic diagram of experimental setup.

TABLE 1. Specifications of lens array (unit: $\mathrm{mm}$ )

\begin{tabular}{l|c|c|c}
\hline \hline & Configuration & Lens pitch & Focal length \\
\hline Array 1 & $13 \times 13$ & $10 \times 10$ & 22 \\
\hline Array 2 & $15 \times 15$ & $5 \times 5$ & 10 \\
\hline Array 3 & $150 \times 150$ & $1 \times 1$ & 3.3 \\
\hline
\end{tabular}




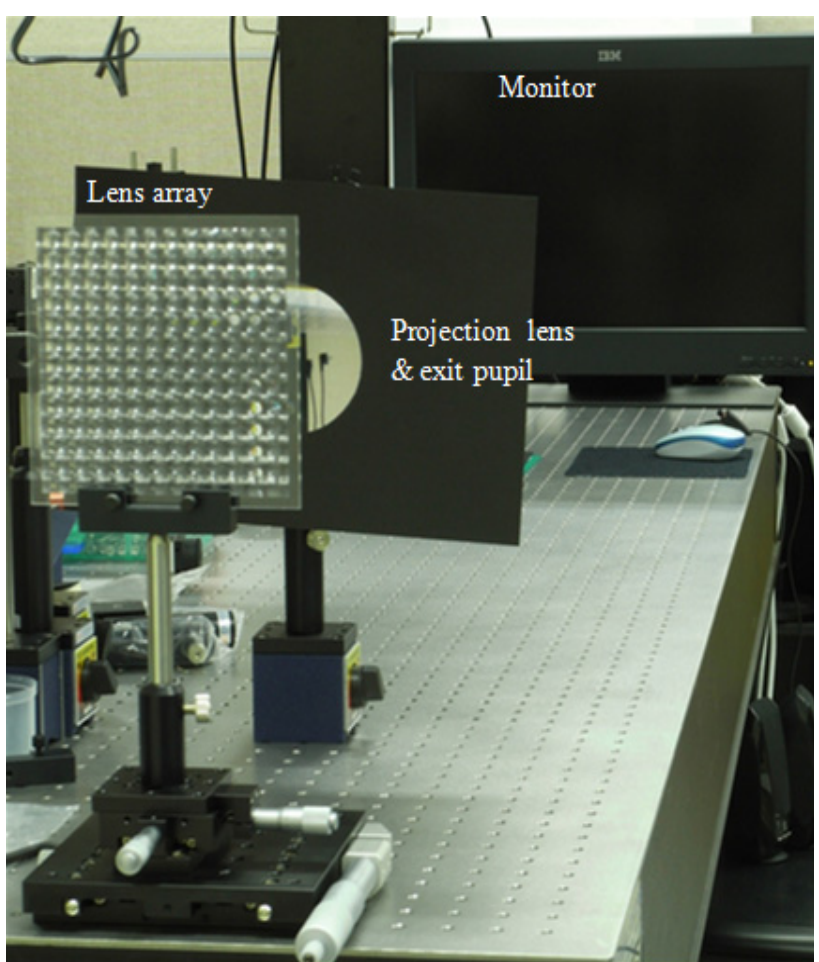

FIG. 6. Experimental setup for visibility analysis of projection-type integral imaging system without diffuser.

lenses are $47 \mathrm{~mm}, 51 \mathrm{~mm}$ and $32 \mathrm{~mm}$, respectively. In order to measure and compare the fill factor with the calculated value, a full white image is displayed on the monitor and a picture is taken from the position of observer. A threshold filter is applied to the picture for easier calculation of fill factor. By counting the white pixels, the fill factor can be obtained. Using this method, for a lens array with $10 \mathrm{~mm}$ elemental lens, the measured overall fill factor is 0.27 and the fill factor of the central lens measures 0.30 which verifies the analysis of Section 2. The small degradation in overall fill factor is due to the shift at the outer elemental lenses, as mentioned before. The shift of a marginal lens is $3.98 \mathrm{~mm}$ both horizontally and vertically. Similarly, for a lens array with $5 \mathrm{~mm}$ and $1 \mathrm{~mm}$ elemental lens, the fill factors of the central lens are 0.29 and 0.30 , while overall fill factors measure 0.27 and 0.25 . The shifts at the marginal lenses are $1.82 \mathrm{~mm}$ and $0.58 \mathrm{~mm}$, respectively.

The objects of letter ' 3 ' and ' $D$ ' were located in the depth plane of $-20 \mathrm{~mm}$ and $-50 \mathrm{~mm}$ from the lens array, respectively. Both letters were integrated in the virtual region. Figure 6 shows the experimental system.

Figure 7 shows the reconstructed image for each case. There are some problems in recognizing letters in the system with the $10 \mathrm{~mm}$ lens array, as shown in Fig. 7. (a), (b) and (c), and the parallax is hard to perceive. Besides, the aberration of the projection lens degrades the image quality. The reconstructed images of the $5 \mathrm{~mm}$ lens array system shown in Fig. 7. (d), (e) and (f) provide higher

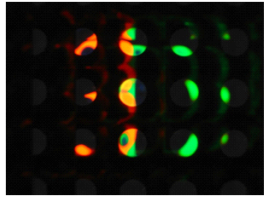

(a) left

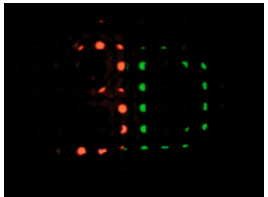

(d) left

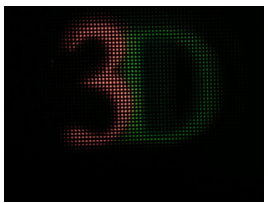

(g) left

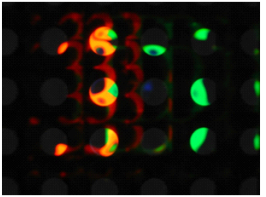

(b) center

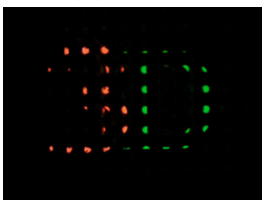

(e) center

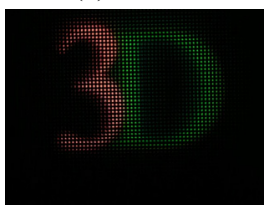

(h) center (c) right

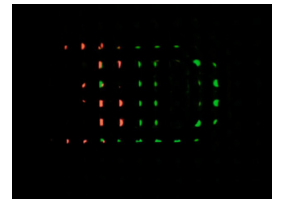

(f) right

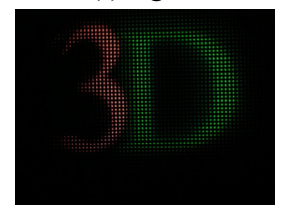

(i) right

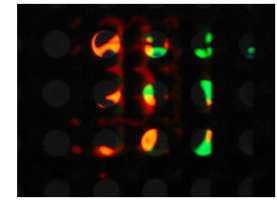

FIG. 7. Comparison of visibility for different configurations of lens array with same fill factor( 0.3$)$. (a) $\sim(\mathrm{c}) 10 \mathrm{~mm}, 6 \times 6$ lens array. (d) (f) $5 \mathrm{~mm}, 12 \times 12$ lens array. (g) (i) $1 \mathrm{~mm}, 60 \times 60$ lens array.

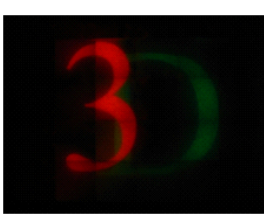

(a)

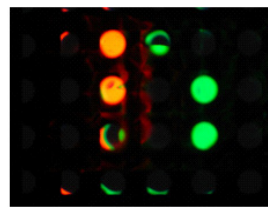

(b)

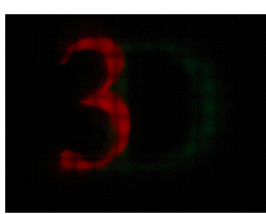

(c)

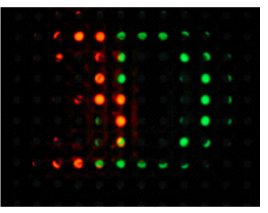

(d)

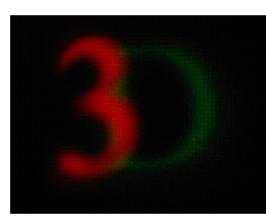

(e)

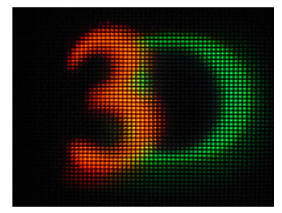

(f)
FIG. 8. Comparison of visibility between projection-type integral imaging system with diffuser (a), (c), and (e) and without diffuser (b), (d), and (f).

visibility than those of the $10 \mathrm{~mm}$ system, but they still show degraded images and poor parallax. On the other hand, there is no problem in recognizing the letters in the $1 \mathrm{~mm}$ lens array system as shown in Fig. 7. (g), (h) and (i). Both letters are clear and the parallax is naturally perceived. Figure 8 shows the integrated images of the projectiontype integral imaging systems with diffuser compared with the systems without diffuser. For the $1 \mathrm{~mm}$ lens array, as shown in Fig. 8, the elemental lens size has little effect on the visibility of the system with and without diffuser

In addition,, as we expected, the system without diffuser shows much brighter images, as shown in Fig. 8. We take the pictures with the same camera settings in order to compare the relative brightness of system. 


\section{DISCUSSION AND CONCLUSIONS}

We have reported a method of evaluating the visibility of reconstructed images in a projection-type integral imaging system without diffuser in terms of the fill factor. If the lens pitch is small enough, the system shows high fill factor and a diffuser is not required. The system without diffuser can improve the optical power efficiency and can solve the problem of restriction in resolution due to the diffusing grain. Also, it provides good image visibility in terms of psychological factors.

However, the pixel number per an elemental lens area determines the 3D effect of the integrated image. Moreover, the diffraction noise which is incurred by small sized lenses can degrade the reconstructed image. Therefore, the elemental lens cannot be reduced below a certain size. Further study for the optimization of the elemental lens size to consider these conditions are necessary and will be performed.

We believe that the proposed analysis can be helpful for designing a projection-type integral imaging system without diffuser as well as other 3D display system applications.

\section{ACKNOWLEDGMENT}

This work was supported by the National Research Foundation of Korea(NRF) grant funded by the Korea government(MEST)(No. 2009-0077704)

\section{REFERENCES}

1. G. Lippmann, "La photographie integrale," Comptes Rendus Acad. Sci. 146, 446-451 (1908).

2. S.-W. Min, J. Kim, and B. Lee, "New characteristic equation of three-dimensional integral imaging system and its applications," Jpn. J. Appl. Phys. 44, L71-L74 (2005).

3. J.-H. Park, S. Jung, H. Choi, and B. Lee, "Integral imaging with multiple image planes using a uniaxial crystal plate," Opt. Exp. 11, 1862-1875 (2003).

4. J. Hong, J.-H. Park, S. Jung, and B. Lee, "Depth-enhanced integral imaging by use of optical path control," Opt. Lett. 29, 1790-1792 (2004).

5. H. Choi, J.-H. Park, J. Hong, and B. Lee, "Depth-enhanced integral imaging with a stepped lens array or a composite lens array for three-dimensional display," Jpn. J. Appl. Phys. 43, 5330-5336 (2004).

6. Y. Kim, J.-H. Park, H. Choi, J. Kim, S.-W. Cho, and B. Lee, "Depth-enhanced three-dimensional integral imaging by use of multilayered display devices," Appl. Opt. 45, 4334-4343 (2006).

7. Y. Kim, H. Choi, J. Kim, S.-W. Cho, Y. Kim, G. Park, and B. Lee, "Depth-enhanced integral imaging display system with electrically variable image planes using polymer-dispersed liquid-crystal layers," Appl. Opt. 46, 3766-3773 (2007).

8. H. Liao, M. Iwahara, N. Hata, and T. Dohi, "High-quality integral videography using a multiprojector," Opt. Exp. 12, 1067-1076 (2004).

9. J. S. Jang, Y. S. Oh, and B. Javidi, "Spatiotemporally multiplexed integral imaging projector for large-scale high-resolution three-dimensional display," Opt. Exp. 12, $557-563$ (2004).

10. J.-S. Jang and B. Javidi, "Three-dimensional projection integral imaging using micro-convex-mirror arrays," Opt. Exp. 12, 1077-1083 (2004).

11. M. Okui, J. Arai, Y. Nojiri, and F. Okano, "Optical screen for direct projection of integral imaging," Appl. Opt. 45, 9132-9139 (2006).

12. R. Martínez-Cuenca, H. Navarro, G. Saavedra, B. Javidi, and M. Martínez-Corral, "Enhanced viewing-angle integral imaging by multiple-axis telecentric relay system," Opt. Exp. 15, 16255-16260 (2007).

13. I. Biederman, "Recognition-by-component theory: a theory of human image understanding," Psychol. Rev. 94, 115-147 (1987).

14. M. J. Tarr, P. Williams, W. G. Hayward, and I. Gauthier, "Three-dimensional object recognition is viewpoint dependent," Nat. Neurosci. 1, 275-277 (1998). 